\title{
Microstructure and Electrical Property of Sputtered Cr-Mo Thin Films
}

\author{
Hideo Murata ${ }^{1}$ and Takuya Ohba $^{2}$ \\ ${ }^{1}$ Metallurgical Research Laboratory, Hitachi Metals Ltd., Yasugi 692-8601, Japan \\ ${ }^{2}$ Department of Materials Science, Shimane University, Matsue 690-8504, Japan
}

\begin{abstract}
Sputter deposited Cr-Mo alloy films have been investigated in electrical properties and microstructures. The resistivity of the films decreased with the Mo concentration from 20 to 40 at $\%$ and increased up to 80 at $\%$ Mo and showed lowest value at Mo film. This resistivity change was discussed with microstructure observations, XRD and recoiled Ar concentration. SEM and TEM images indicated that several grains were combined and form lager aggregated grains and columnar structure. Stress in the films was discussed also with relationship of Mo concentration. [doi:10.2320/matertrans.MRP2008206]
\end{abstract}

(Received July 4, 2008; Accepted September 29, 2008; Published November 25, 2008)

Keywords: sputtering film, chromium molybdenum alloy, resistivity, internal stress, microstructure, lattice constant

\section{Introduction}

Sputtered deposition method is one of physical vapor depositions (PVD) for making thin films. The method has several advantages in comparison with other PVD. Firstly, the method is adaptable to widely materials such as high melting-point metals, oxide, organic materials and alloys. Secondary, the method deposits materials rather uniformly with large area substrates. Therefore, the sputtering method has been used for practical applications such as surface treatment, optical control films and thin film electron device, which support information industry.

Sputtered Cr films have high adhesion to substance and high erosion resistance. In addition, $\mathrm{Cr}$ films react with various gases easily. Reactive sputtered $\mathrm{Cr}$-nitride and $\mathrm{Cr}$ alloy-nitride films have high hardness, and were widely used in the application of hard coating. ${ }^{1,2)}$ Reactive sputtered $\mathrm{Cr}$ oxide films have low reflectivity, and thus were used photomask for semiconductor and color filter for liquid crystal display (LCD). ${ }^{3,4)}$ Sputtered $\mathrm{Cr}$ films have been widely utilized in industrial use for its high reflectivity, high conductivity and good optical properties. The $\mathrm{Cr}$ films were used for underlayer of magnetic recording media in rigid disk drive. ${ }^{5,6)}$ Recently, the Cr alloy films, whose lattice constants and/or microstructures were controlled by alloying, have been studied for developing a possibility of Cr films. ${ }^{7)}$ These films were used for conductivity films of gate line on the thin film transistor (TFT) of LCD. ${ }^{8,9)}$ Those sputtered $\mathrm{Cr}$ film is required low resisitivity and low stress for large area substrate. ${ }^{10)}$ For producing low resistivity and low stress $\mathrm{Cr}$ films, the sputtering condition is low Ar pressure, high input power on targets and high temperature of the substrate. ${ }^{11)}$ Bias sputtering method is one of useful methods for producing low stress $\mathrm{Cr}$ films, and is known to be peening. ${ }^{12)}$ However the bias sputtering method introduces lattice distortion and increases the resistivity. ${ }^{13)}$

There is few paper on the resistivity and a stress of $\mathrm{Cr}$ alloy films. In this paper, the effects on alloying of $\mathrm{Cr}$ films were examined. Mo was chosen for alloying element. Because the resistivity of Mo is lower than $\mathrm{Cr}$, and the stress of sputtered Mo film were lower than that of the $\mathrm{Cr}$ film on the same sputtering condition, in general. Further, Mo belongs to
Group VIB, which is the same as $\mathrm{Cr}$, and forms a solid solution in the phase diagram. The electrical properties such as resistivity of Cr-Mo films were investigated with relationship of microstructure and internal stress.

\section{Experimental}

The Cr-Mo alloy films were deposited by a DC magnetron sputtering system on Corning 7059 glass substrates and 4 inch Si (110) wafers. The sputtering system consists of a load-locked chamber and the main deposition chambers with the cryopump. The vacuum reaches under $5 \times 10^{-6} \mathrm{~Pa}$. The deposition condition of the films was fixed as follows; pre-heating of $150^{\circ} \mathrm{C}$ by an infrared lamp heater, with input power on target of $1500 \mathrm{~W}\left(7 \mathrm{~W} / \mathrm{cm}^{2}\right)$ and Ar gas pressure of $0.5 \mathrm{~Pa}$. Thicknesses of the films deposited were $200 \mathrm{~nm}$, which was controlled by deposition time. Resistivities of the films were measured with four terminal method (Mitsubishi Chemical Co. Loresta AP MCP-T400). Internal stresses of the films were estimated from the curvature of $\mathrm{Si}$ wafer with thermal treatment (Toho Technology Co. FLX-2320). The concentration of the films was determined by electron probe X-ray microanalysis (EPMA Nihon Denshi Co. JXR8900R). The crystal structures of the films were investigated by X-ray diffraction with $\mathrm{CuK} \alpha$ radiation (Rigaku RINT-2500). The surface and cross-section morphology of the films were observed by field-emission scanning electron microscope (FE-SEM Hitachi Ltd. S-4000). Microstructures of the films were observed by transmission electron microscope (TEM Hitachi Ltd. H-700). The composition of Cr-Mo alloy films were changed by using different target materials, whose Mo content was 20, 40, 60 and 80 at\% on this study. The physical properties of $\mathrm{Cr}$ and $\mathrm{Mo}$ were shown in Table 1. ${ }^{14,15)}$

Table 1 Physical properties of $\mathrm{Cr}$ and Mo.

\begin{tabular}{cccccc}
\hline Materials & $\begin{array}{c}\text { Atomic } \\
\text { weight }\end{array}$ & $\begin{array}{c}\text { Density } \\
\left(\mathrm{Mg} / \mathrm{m}^{3}\right)\end{array}$ & $\begin{array}{c}\text { Lattice constant } \\
(\mathrm{nm})\end{array}$ & $\begin{array}{c}\text { Resistivity } \\
\left(10^{-8} \Omega \mathrm{m}\right)\end{array}$ & $\begin{array}{c}\text { Sputtering } \\
\text { rate }(-)\end{array}$ \\
\hline $\mathrm{Cr}$ & 52.00 & 7.19 & 0.288 & 12.7 & 1.35 \\
$\mathrm{Mo}$ & 95.94 & 10.22 & 0.314 & 5.2 & 0.90 \\
\hline
\end{tabular}


Table 2 Mo concentration of Target materials and films deposited. Mo concentrations of the films were good agreement in the concentrations of target materials.

\begin{tabular}{lllll}
\hline Mo concentration of Target (at\%) & 20 & 40 & 60 & 80 \\
\hline Mo concentration of film (at\%) & 21 & 42 & 63 & 81 \\
\hline
\end{tabular}

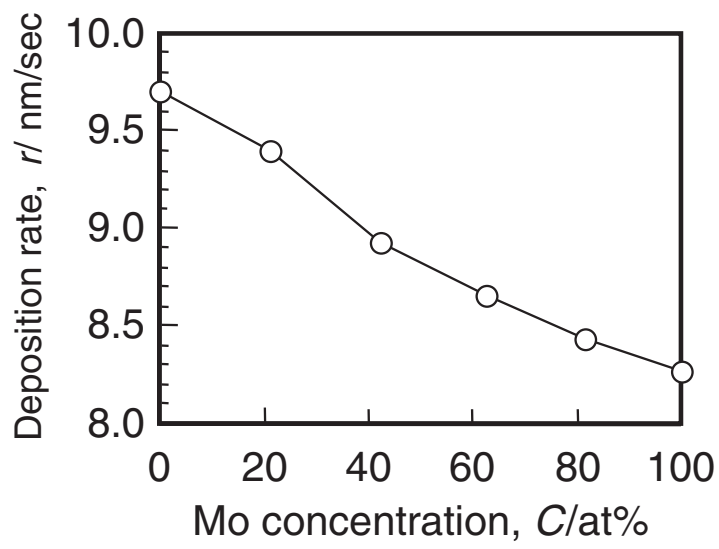

Fig. 1 Deposition rates of $\mathrm{Cr}-\mathrm{Mo}$ films against Mo concentrations. Deposition rate decreases monotonically with increasing Mo concentration.

\section{Results}

\subsection{Concentration of the film}

Concentrations of the target materials and Cr-Mo films produced were shown in Table 2 . Fairly good agreement in the concentrations shown here reflects advantage of sputtering method.

Figure 1 shows deposition rates of $\mathrm{Cr}$-Mo film against Mo concentrations. The deposition rate decreases with increasing Mo concentration. Lower sputtering rate of Mo shown in Table 1 causes the low deposition rate at higher Mo concentration.

\subsection{Resistivity}

Mo concentration dependence of the resistivity on Cr-Mo alloy films is shown in Fig. 2. Resistivities of the films become lower in the range of 20-40 at\%Mo compared with $\mathrm{Cr}$ film, and become the same level around 60 at\% Mo, and become a little bit higher at 80 at $\% \mathrm{Mo}$, and then become lowest at Mo film. Resistivity of binary solid solution, whose elements belong to the same group in the periodic table, in general, shows Nordheim's rule, that is, risistivity shows approximately parabolic shape with maximum value at about equi-atomic composition. ${ }^{16)}$ It is reported that resistivity of bulk in Cr-Mo was measured and followed the rule up to 8 at\%Mo. ${ }^{17)}$ Resistivity being assumed the Nordheim's rule is shown with dotted line in Fig. 2, whose both ends (Mo and $\mathrm{Cr}$ ) were assumed to be bulk values in Table 1 and the value at 8 at\% Mo was assumed to be that of bulk measurement in reference 17). Comparison with resistivity of bulk pure metals makes us understand that the resistivity of the films indicate two times higher in $\mathrm{Cr}$ film and three times higher in Mo film. Higher resistivity of the films was caused by several kinds of defects in the film and surface. Although we did not

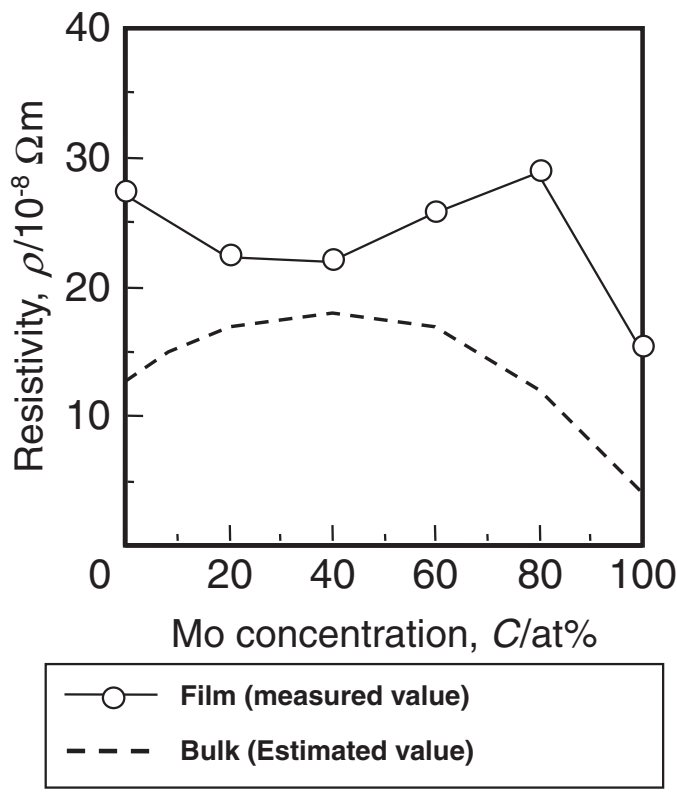

Fig. 2 Mo concentration dependence of resisitivity on Cr-Mo films. The resistivity of the film decreases up to 40 at $\% \mathrm{Mo}$ and increases up to 80 at\% Mo. The lowest resistivity was obtained in Mo film.

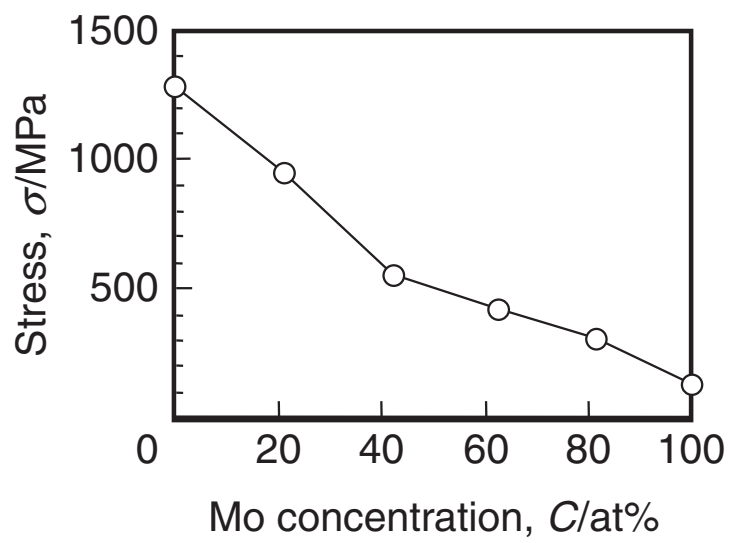

Fig. 3 Mo concentration dependence of the stress on Cr-Mo films. The stress decreases monotonically with increasing Mo concentration.

measure the resistivity within the range of $10 \mathrm{at} \% \mathrm{Mo}$, the decrease of the resisitivity of Cr-Mo films in the range of 20 and 60 at\% Mo looks curious.

\subsection{Stress of the film}

The stress of the sputtered film was governed by internal stress, which depends on sputtering condition. ${ }^{18)}$ Mo concentration dependence of the stress in the Cr-Mo alloy films is shown in Fig. 3. The stress of $\mathrm{Cr}$ film is large tensile stress and that of alloy films become lower with increasing of Mo concentration. Thus the alloying $\mathrm{Cr}$ with Mo lowers stress of the $\mathrm{Cr}$ film.

\subsection{Structure of the films}

XRD patterns were measured for prepared alloy films and indicate that crystal structure of all alloy films are bcc with strongly preferred-oriented (110). Preferred orientation of (110) is quite general because close packed planes are 

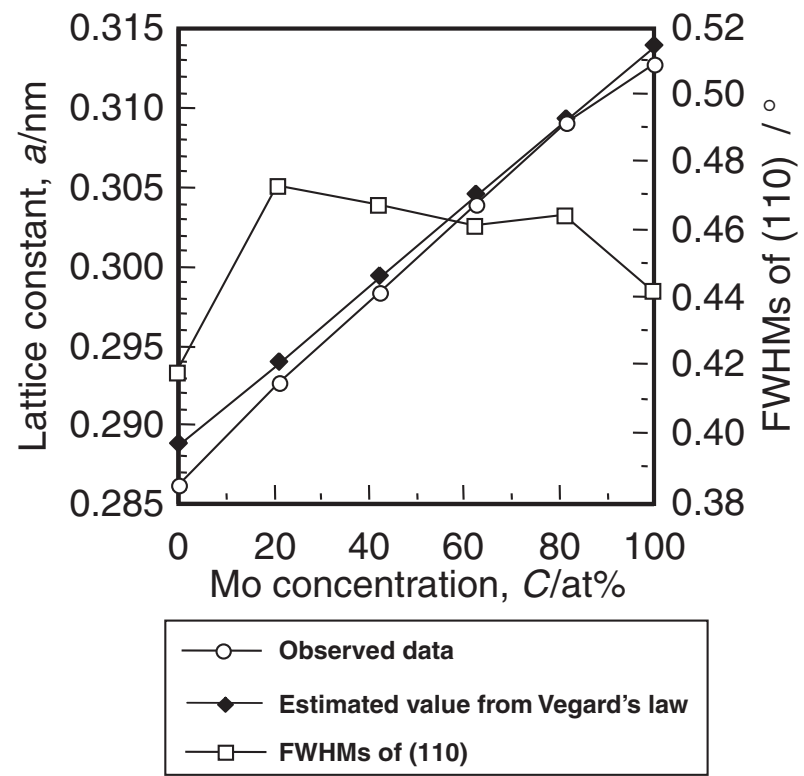

Fig. 4 Mo concentration dependence of lattice constant on Cr-Mo films. The lattice constants follow Vegard's law.

stacked while deposition, in general, that is, (110) of bcc was preferably oriented. ${ }^{19)}$

Lattice constants for the films were determined using (110) reflection by XRD. These data were compared with lattice constants obtained from Vegard's law. ${ }^{20)}$ Lattice constants for $\mathrm{Cr}$ and Mo were refereed from bulk data. Mo concentration dependence of the lattice constants is shown in Fig. 4, which shows that observed values are smaller than calculated values. The differences between observed values and calculation values decrease with Mo concentration from 20 to 80 at\% and increase again at Mo. In all concentration range, observed values are smaller than calculated values as mentioned above. Thus the residual tensile stress may be applied. FWHMs of (110) peak on Cr-Mo alloy films shown in Fig. 4 are broader than those of $\mathrm{Cr}$ and Mo films and indicate that Cr-Mo alloy films include strain. Expansion of the lattice constant with increase of Mo concentration reflects that larger atomic radius atoms, Mo atoms, were replaced with $\mathrm{Cr}$ atoms.

\subsection{SEM and TEM Observations of Cr-Mo films}

FE-SEM images of Cr-Mo films were shown in Fig. 5. The observations were carried out from $45^{\circ}$ of the film surface normal. Thus the surface and cross-section are observed simultaneously. The area of observed point was focused on edge of the surface and cross-section. The structures of films are columnar and have surface relief along the column. The sizes of the columns in Cr films are approximately 50-60 nm and those of column in Mo films are smaller. The sizes of columns in Cr-Mo films increase with Mo concentration up to 60 at $\%$ Mo films. Boundaries between columns become diffuse. The sizes of columns in 80 at $\%$ Mo film are finer and similar to those of column in Mo film.

TEM images of films are shown in Fig. 6. Black and white tones represent crystal orientation difference. Grain sizes were estimated here to be small round shapes with same tone area. Grain sizes of $\mathrm{Cr}$ films are $25 \mathrm{~nm}$ and those of Mo films

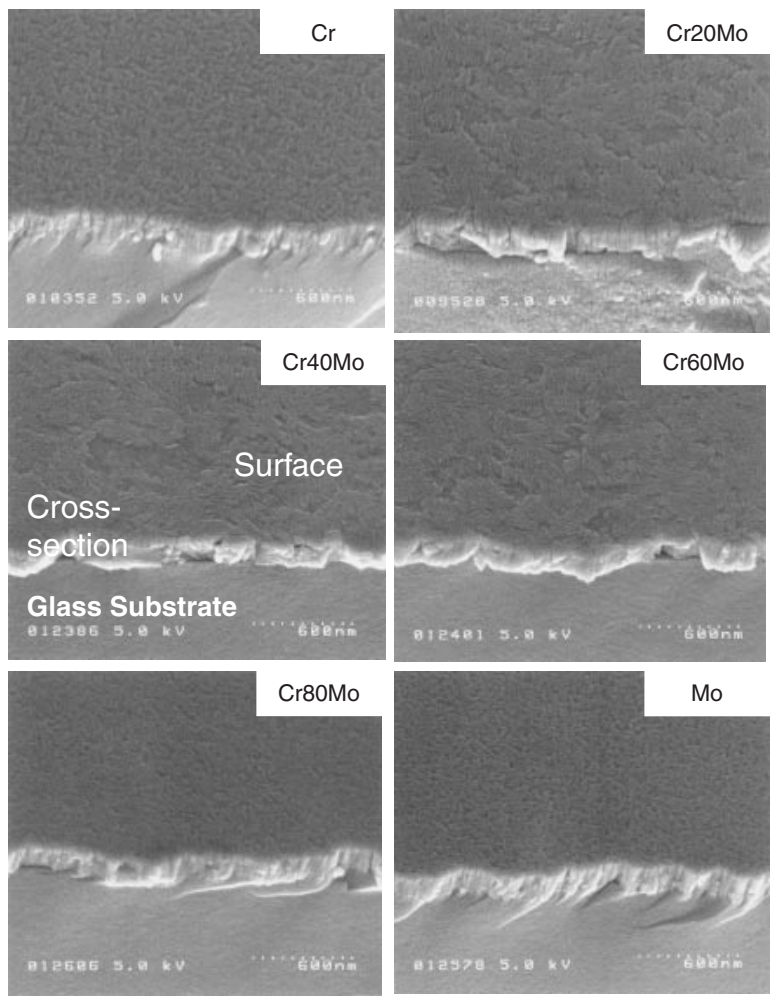

Fig. 5 FE-SEM images of Cr-Mo films. The structures of films are columnar. The sizes of the columns are changes according to Mo concentration.
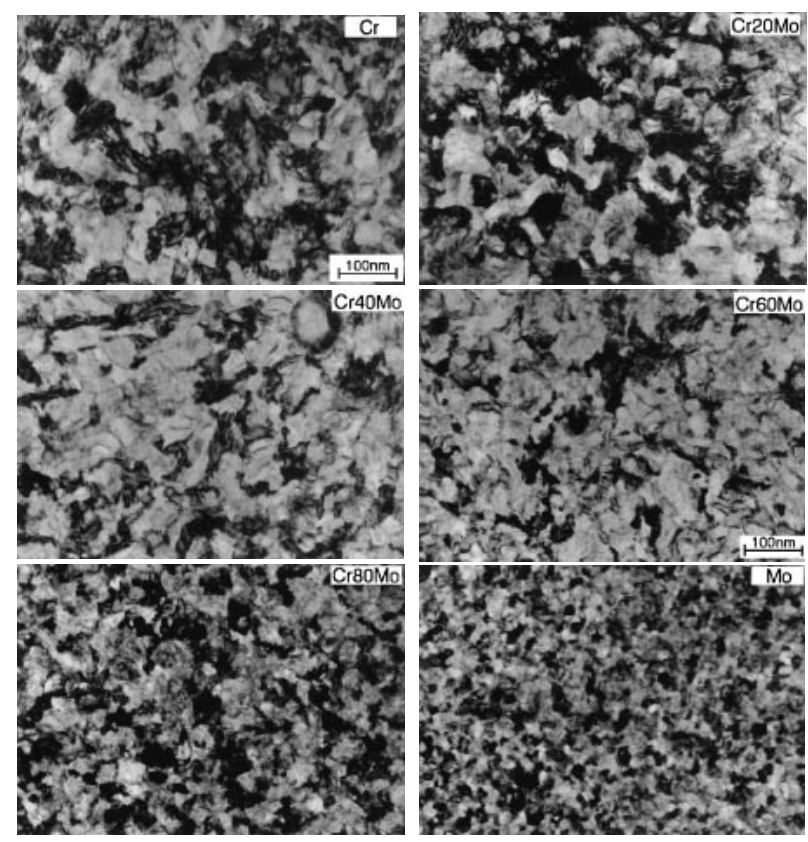

Fig. 6 TEM images of Cr-Mo films. The grain structures of films look like gathering the several grains. The grain structures change according to Mo concentration.

are $15 \mathrm{~nm}$. The grain sizes of Cr-Mo film shown in Fig. 7 with open circles are almost constant up to 40 at\% Mo and decreases monotonically to Mo film. Further characteristics can be seen, that is, the grain structure of films looks like gathering the several grains. These grains formed the 


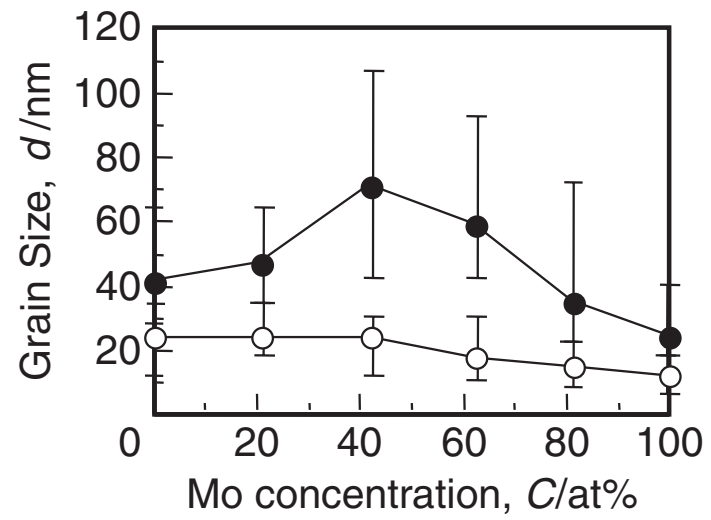

- - Single Grain

- Aggregated grain

Fig. 7 Grain sizes of Cr-Mo films against Mo concentrations. The aggregated grain sizes increase up to 40 at $\%$ Mo and decrease gradually to Mo.

aggregated grain, whose sizes were estimated to be one of nearly tone. Those aggregated grain sizes are also shown in Fig. 7 with filled circles. The aggregated grain sizes increases up to $70 \mathrm{~nm}$ at 40 at $\%$ Mo and decreases gradually to $25 \mathrm{~nm}$ at Mo film. The sizes of columns in SEM observations were almost the same as aggregated grain sizes rather than the single grain sizes. Thus, the columns of films are composed of combined several grains.

\section{Discussion}

Structure of the films depend on conditions of sputtering such as substrate temperature, pressure of Ar, incident power of target, in general, and also depend on melting points of materials. The deposition conditions in this report are the same for all alloy films. It is well known that structure of films is classified into ZONE-1, ZONE-T, ZONE-2 and ZONE-3 depending on Ar pressure and the ratio of substrate temperature and melting point of the material $\left(T_{\mathrm{s}} / T_{\mathrm{M}}\right){ }^{21)}$ $T_{\mathrm{S}} / T_{\mathrm{M}}$ of $\mathrm{Cr}$ film is 0.2 and that of Mo film is 0.15 . The microstructure of $\mathrm{Cr}$, Mo, Cr-Mo alloy films look like ZONE-1 or ZONE-T from the cross-section view of Fig. 5 . Melting points are considered for explaining the microstructure. Grain sizes of Mo films are smaller than those of $\mathrm{Cr}$ films, corresponding to melting points; that is, melting point of Mo is higher than that of $\mathrm{Cr}$. While grain sizes of $\mathrm{Cr}-\mathrm{Mo}$ films are in between that of $\mathrm{Cr}$ films and Mo films as was indicated above, the aggregated grain sizes of $\mathrm{Cr}-\mathrm{Mo}$ films are not in between that of $\mathrm{Cr}$ and Mo. Thus the process of forming the aggregated grain is considered below.

An effect of recoiled Ar, which is characteristics of sputtering method, is considered. Recoiled Ar atoms or ions are deposited on the films. It is reported that amount of recoiled Ar increase for heavy atoms deposition. ${ }^{22)}$ Amount of Ar in the films was measured by EPMA and shown in Fig. 8. Amount of $\mathrm{Ar}$ increases with increase of Mo concentration, which follows the tendency mentioned above. Exception is found at Mo film, that is, amount of Ar in Mo films decreases. The Ar atoms were trapped in interstitial

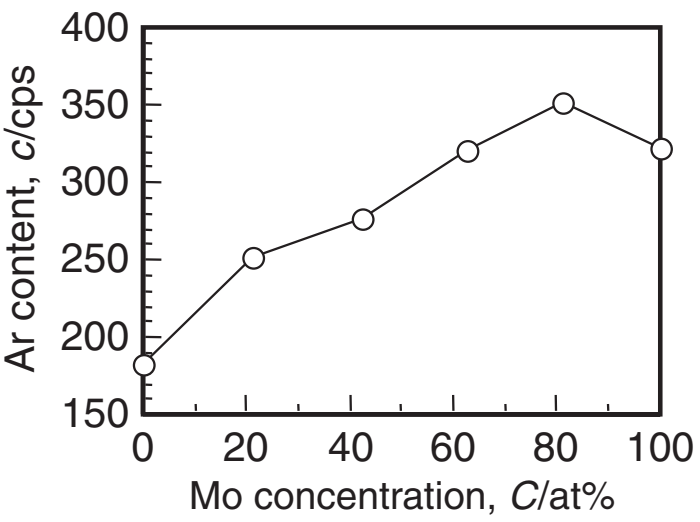

Fig. 8 Ar contents of Cr-Mo films against Mo concentrations. The amount of $\mathrm{Ar}$ increases with increasing Mo concentration.

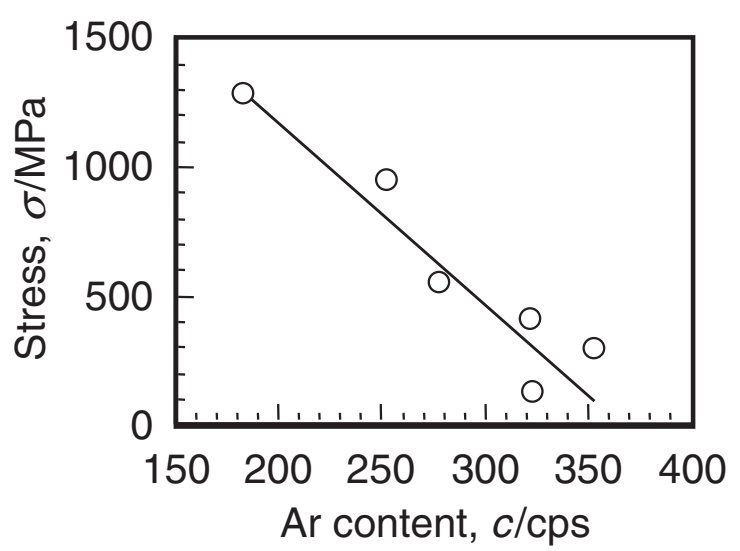

Fig. 9 The stress of Cr-Mo films as a function of Ar content. The stress of the film decreases with increasing Ar content.

sites of the film. Thus, the many Ar atoms had been taken in the alloy films easier than pure metal films. Recoiled $\mathrm{Ar}$ atoms release heat in the film, and released heat give the energy to atoms and make a rearrangement of atoms around boundaries. Thus wider grain boundary gaps become narrower than original one. This is the process of being produced aggregated grain.

Recoiled Ar atoms change the structure of $\mathrm{Cr}-\mathrm{Mo}$ film from ZONE-1 to ZONE-T. Thus the strain of the structure was relaxed, and the relaxation of the structure decreases defects. Figure 9 shows the stress of Cr-Mo film against amount of Ar. The stress of the films decreases with increase of $\mathrm{Ar}$ content. It is considered that the peening effect of recoiled Ar is similar to the bias sputtering method.

Vegard's law indicated that Mo atoms are replaced with $\mathrm{Cr}$ atoms by alloying. Replacements of Mo atoms by $\mathrm{Cr}$ make a possibility of relaxation of tensile stress. In the present case, however, Ar atoms must be the main reason of the stress relaxation as shown in Fig. 9. Where do Ar atoms locate or how to relax the stress? The recoiled Ar atoms assist rearrangement of grain boundaries creating aggregated grains. Those Ar atoms must be captured and located around boundaries. Location of $\mathrm{Ar}$ atoms around boundaries gives compressive force between aggregated grains and causes a decrease of tensile stress except for Mo film. 
Resistivity is caused by electron scattering with crystal defects such as impurities, grain boundaries. Grain sizes in bulk are larger than the mean free path of the electron and are less influence on the resistivity. $\mathrm{Cr}$ and Mo belong to the same Group VIB, and Cr-Mo alloy forms a solid solution in the phase diagram. The resistivities of Cr-Mo alloy films are affected by crystal defects. The aggregated grain sizes in the present films showed a correlation with resistivities, that is, larger aggregated grain sizes showed lower resistivity as shown in Fig. 6 and Fig. 7. Mean free path of electron in $\mathrm{Cr}$ is a few ten $\mathrm{nm}$. The aggregated grain may work as a larger single grain. Aggregated grain sizes of $\mathrm{Cr}-\mathrm{Mo}$ alloy films were from 15 to $70 \mathrm{~nm}$. Thus the main reason of resistivity is the aggregated grain sizes in films, which are comparable with the mean free path of electron.

Recoiled Ar atoms must play an important role in $\mathrm{Cr}-\mathrm{Mo}$ alloy films. However exception was found at Mo film. Further study is necessary to understand the phenomena found in Cr-Mo film completely.

\section{Conclusions}

Sputtered Cr-Mo alloy flims were investigated, which forms a solid solution belonging to the same Group VIB in the phase diagram.

(1) Lattice constants of alloy films were determined by XRD and showed a little bit smaller than the values from Vegard's law. FWHMs of (110) reflection on the alloy films were broader than that of $\mathrm{Cr}$ and $\mathrm{Mo}$ film.

(2) Resistivity against Mo concentration was obtained. Resistivities of alloy films (20 and 40 at\%Mo) show lower resistivity than $\mathrm{Cr}$ film.

(3) SEM and TEM images indicated that several grains were combined and form lager aggregated grains and columnar structure.

(4) Resistivity was strongly affected by aggregated grain sizes of films. Especially, resistivities between 20 and 60 at\% Mo decrease because of aggregated gain sizes increase in this range.

(5) Mo concentration dependence of the stress was determined. Higher tensile stress at $\mathrm{Cr}$ film decreased monotonically with increase of Mo concentration.

(6) The stress of films depends on amount of Ar. Recoiled
Ar atoms must play an important role for the above sputtering behavior in Cr-Mo alloy films.

\section{Acknowledgement}

The authors are grateful for the experimental support from Metallurgical Research Laboratory of Hitachi Metals Ltd., Japan.

\section{REFERENCES}

1) E. Ando and S. Suzuki: J. Non-Cryst. Solid 218 (1977) 68-73.

2) K. K. Ho, C. E. Yong, H. S. Gyun, P. B. Gyu and Y. J. Hong: Surf. Coat. Technol. 201 (2006) 4068-4072.

3) L. C. Hsia: J. Electrochem. Soc. 131 (1984) 2133-2137.

4) T. Takahashi: Electron. Parts Mater. extra number of July (2001) 62-68.

5) T. Yamada, N. Tani, M. Ishikawa, Y. Ota, K. Nakamura and A. Itoh: IEEE Trans. Magn. MAG-21 (1985) 1429-1431.

6) J. C. Allan and R. D. Fisher: IEEE Trans. Magn. MAG-23 (1987) 122-124.

7) M. A. Parker, J. K. Howard, R. Ahlert and K. R. Coffey: J. Appl. Phys. 73 2A (1993) 5560-5562.

8) M. Hayama: Semicond. World 7 (1991) 176-178 (in Japanese).

9) H. Tanaka: SID 1987 Digest (1987) pp 140.

10) ANELVA C-3900 series: Semicond. FPD World 22 (2003) 150 (in Japanese).

11) H. Murata: Hitachi-Metals Technology 12 (1996) 23-28 (in Japanese).

12) D. W. Hoffman and J. A. Thornton: Thin Solid Films 40 (1977) 355-363.

13) G. C. A. M. Janssen and J. D. Kamminga: Appl. Phys. Lett. 85 (2004) 3086-3088.

14) Metal data book 4th revised edition, (The Japan Inst. Metals: 2004) pp. 1-40 (in Japanese).

15) R. V. Stuart (Translated by M. Mouri and A. Kazusaka): Vacuum Techinology, Thin Films, and Sputtering, (GIHODO SHUPPAN Co.Ltd) pp. 112 (in Japanese).

16) The theory of the properties of Metals and Alloys, ed. by N. F. Mott and H. Jones, (Dover Publications, New York, 1958) 296-298.

17) S. Arajs, E. E. Anderson and K. V. Rao: J. Less-Common Metals 26 (1971) 157-164.

18) D. W. Hoffman and J. A. Thornton: J. Vac. Sci. T. 20 (1982) 355-358.

19) A. Kinbara: SPUTTERING, (University of Tokyo Press. 1984) pp. 181 (in Japanese).

20) C. Barrett and T. B. Massalski: Structure of Metals 3rd Revised Edition Pergamon, (1980) pp. 372.

21) J. A. Thornton: J. Vac. Sci. T. 11 (1974) 666-670.

22) J. A. Thornton, J. Tabock and D. W. Hoffman: Thin Solid Films 64 (1979) 111-119. 